\title{
LETTER
}

Myelodysplastic syndromes

\section{Impact of somatic mutations on response to lenalidomide in lower-risk non-del(5q) myelodysplastic syndromes patients}

\author{
Valeria Santini $\mathbb{1}^{1}$ - Pierre Fenaux ${ }^{2}$ - Aristoteles Giagounidis ${ }^{3}$ - Uwe Platzbecker ${ }^{4}$ Alan F. List ${ }^{5} \cdot$ Torsten Haferlach $^{6}$.

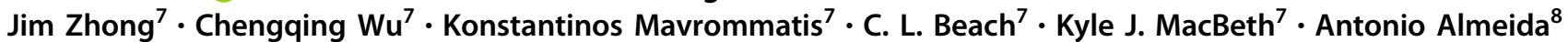

Received: 2 March 2020 / Revised: 29 May 2020 / Accepted: 1 July 2020 / Published online: 13 July 2020

(c) The Author(s) 2020. This article is published with open access

\section{To the Editor}

Myelodysplastic syndromes (MDS) are bone marrow neoplasias that involve mutations in genes affecting various cell pathways [1]. Both clonal evolution and the type of somatic mutations appear to be associated with response to treatment $[1,2]$. Response to hypomethylating agents possibly correlates with mutations in TET2 [3], TP53 [4], and mutation burden [5]. Erythroid response to erythropoiesisstimulating agents (ESAs) has also been linked to mutation burden [6]. Recently, luspatercept has been shown to enhance late-stage erythropoiesis in non-del(5q) MDS with ring sideroblasts regardless of mutational status [7].

Lenalidomide therapy has previously been investigated in MDS-005 (NCT01029262); a randomized, phase 3 trial of ESA-refractory or -ineligible patients with lower-risk

Supplementary information The online version of this article (https:// doi.org/10.1038/s41375-020-0961-3) contains supplementary material, which is available to authorized users.

Valeria Santini

valeria.santini@unifi.it

1 MDS UNIT, Hematology, Azienda Ospedaliero Universitaria Careggi, University of Florence, Florence, Italy

2 Service d'Hématologie Séniors, Hôpital Saint-Louis, Université Paris 7, Paris, France

3 Department of Oncology, Hematology, and Palliative Care, Marien Hospital Düsseldorf, Düsseldorf, Germany

4 Medical Clinic and Policlinic I, Hematology and Cellular Therapy, Leipzig University Hospital, Leipzig, Germany

5 Formerly Department of Malignant Hematology, H. Lee Moffitt Cancer Center and Research Institute, Tampa, FL, USA

6 MLL Munich Leukemia Laboratory, Munich, Germany

7 Bristol Myers Squibb, Princeton, NJ, USA

8 Instituto Português de Oncologia de Lisboa Francisco Gentil, Lisbon, Portugal non-del(5q) MDS [8]. Previous studies investigating markers of response to lenalidomide, alone or in combination with ESAs, lacked statistical power and require independent validation $[9,10]$. We carried out an exploratory investigation into the relationship between gene mutations and response to lenalidomide in patients from the MDS005 study.

Efficacy endpoints from the MDS-005 trial were analyzed according to gene mutation status [11]. Gene expression analysis was performed at baseline as a primary endpoint of the MDS-005 study, other analyses were exploratory endpoints. DNA was isolated from bone marrow mononuclear cells $(n=177)$ or whole blood $(n=21)$ collected at screening from consenting patients with adequate tissue for exploratory biomarker analysis. Targeted next-generation sequencing of 56 genes was performed at the Munich Leukemia Laboratory (Supplementary Table 1).

Baseline characteristics of the biomarker cohort $(N=198$; 130/68 received lenalidomide/placebo) were generally comparable to those of patients not included (Supplementary Fig. 1; Supplementary Table 2). Within the biomarker cohort, baseline characteristics were well balanced between patients receiving lenalidomide and those receiving placebo (Supplementary Table 3). Baseline characteristics of biomarker cohort patients by ASXL1 mutation status are shown in Supplementary Table 4. In total, 173 (87.4\%) harbored at least 1 mutation in the 56 genes assessed (Supplementary Table 5). Fifty-five patients (27.8\%) had a single mutation, $63(31.8 \%)$ had 2 mutations, and 55 (27.8\%) had >2 mutations. Mutations were detected in 30 of the 56 genes assessed (Supplementary Fig. 2a). The most commonly observed gene mutations were SF3B1 (58.6\% of patients), TET2 (33.0\%), ASXL1 (23.2\%), and DNMT3A (13.6\%). The most frequently observed co-mutations were SF3B1 and TET2 (22.7\%), SF3B1 and DNMT3A (10.1\%), SF3B1 and ASXL1 (9.6\%), and TET2 and ASXL1 (8.6\%) (Supplementary Fig. 2b); $99.1 \%$ of patients with $S F 3 B 1$ mutations had ring sideroblasts $\geq 5 \%$ (Supplementary Table 6). The relationship 

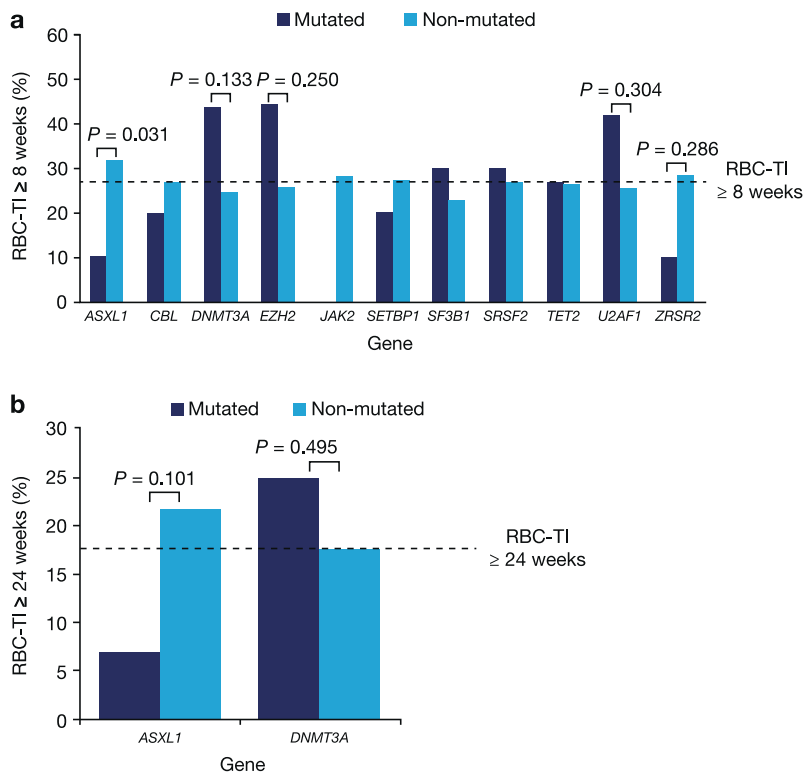

Fig. 1 Proportion of patients treated with lenalidomide who achieved RBC-TI according to gene mutation status. RBC$\mathrm{TI} \geq 8$ weeks (a) and $\geq 24$ weeks (b). Dotted lines represent response rates in all patients treated with lenalidomide $(26.9 \%$ and $17.5 \%$ for RBC-TI $\geq 8$ and $\geq 24$ weeks, respectively). RBC-TI red blood cell transfusion independence.

between mutation status and cytogenetic abnormalities at baseline is shown in Supplementary Table 7.

Among lenalidomide-treated patients, the proportion of patients achieving red blood cell transfusion independence (RBC-TI) $\geq 8$ weeks was significantly lower in those with ASXL1 mutations than those without (10.3\% vs. $31.7 \%$; $p=$ 0.031) (Fig. 1a). The proportion of patients achieving RBC$\mathrm{TI} \geq 8$ weeks was nominally higher in those with $D N M T 3 A$ mutations than in those without $(43.8 \%$ vs. $24.6 \% ; p=$ 0.133 ). RBC-TI $\geq 8$ weeks was achieved by $42.9 \%$ of patients with both DNMT3A and SF3B1 mutations (which co-occurred in most patients), 1 of 2 patients (50.0\%) with DNMT3A only, $27.1 \%$ of patients with $S F 3 B 1$ only, and $21.8 \%$ of patients with neither DNMT3A nor $S F 3 B 1$ mutations. Eleven patients (5.6\%) had $E Z H 2$ mutations; of the 9 receiving lenalidomide, $4(44.4 \%)$ achieved RBC-TI $\geq 8$ weeks, versus 31 of 121 (25.6\%) patients without EZH2 mutations $(p=0.250)$ (Fig. 1a). Mutations in other genes were not significantly associated with response to lenalidomide.

No specific single mutation was associated with achievement of $\mathrm{RBC}-\mathrm{TI} \geq 24$ weeks with lenalidomide, although patients with $A S X L 1$ mutations had a nominally lower rate of RBC-TI $\geq 24$ weeks than those without ASXL1 mutations $(6.9 \%$ vs. $21.8 \%$; $p=0.101)$ (Fig. 1b). Mutation status of the 12 patients who achieved RBC-TI $\geq 52$ weeks is shown in Supplementary Table 8 . There was no association between mutation status and duration of RBC-TI in patients treated with lenalidomide.
The number of mutations present did not appear to correlate with response, and no relationship was found between the variant allele frequency of mutations and response rates (Supplementary Fig. 3). Of 27 lenalidomide-treated patients evaluable for cytogenetic response, $5(18.5 \%)$ achieved cytogenetic complete response and $4(14.8 \%)$ had cytogenetic partial response (PR) (Mutations status: Supplementary Table 9). Presence of an A/G polymorphism at rs1672753 in the CRBN gene was not associated with response in the MDS-005 study population. No patients evaluated in our study had a CSNKIAl mutation.

Overall survival (OS) by treatment group and ASXL1 mutation status is shown in Fig. 2a. Mutations in the ASXL1 gene were associated with significantly shorter median OS, regardless of treatment group (log-rank $p<$ 0.0001). Among patients with ASXL1 mutations, median OS was 2.0 years (95\% confidence interval (CI) 1.5-3.1) with lenalidomide and 2.1 years $(95 \%$ CI 0.9 -not estimable (NE)) with placebo $(p=0.576)$. Shorter OS in patients with ASXL1 mutations was confirmed with a Cox proportional hazards model (hazard ratio (HR) 2.6 [95\% CI 1.5-4.6]) controlling for clinical covariates including baseline erythropoietin (EPO), blast percentage, and Revised International Prognostic Scoring System (IPSSR) risk. The presence of DNMT3A mutations had no significant effect on OS in patients receiving placebo (logrank $p=0.3228$ ) (Fig. 2b); however, there was a trend toward longer median OS with lenalidomide (NE [95\% CI 2.0 years-NE]) compared with placebo (1.6 years [95\% CI 0.6-NE]) $(p=0.123)$. Mutations in SF3B1 and TET2 had no significant effect on OS.

The presence of a mutation in any of 5 genes previously linked to poor prognosis (that is ASXL1, ETV6, EZH2, $R U N X 1$, and TP53) [12] was associated with shorter median OS (log-rank $p=0.0018$ ) (Fig. 2c). Median OS was 2.1 years (range 1.7-3.1) in lenalidomide-treated patients with mutations in any of the 5 genes versus NE (NE-NE) in nonmutated lenalidomide-treated patients $(p=0.0003)$.

In patients receiving lenalidomide, a higher number of mutations was significantly associated with shorter median OS (log-rank $p=0.0005$ ) (Fig. 2d). Median OS was NE in patients with 0-3 mutations and 1.7 years (range 0.6-NE) in patients with $\geq 4$ mutations.

Patients with $\geq 4$ mutations were more likely to have a high serum EPO level $(>500 \mathrm{mU} / \mathrm{ml})$ than patients with fewer mutations $(p=0.0190)$. Int-1-risk patients with high EPO levels were more likely to have $\geq 4$ mutations than those with low EPO levels (10/16 patients [62.5\%] versus 6/ 16 patients [37.5\%]; $p=0.0962)$; there was no significant difference for Low-risk patients (3/6 patients [50.0\%] vs. 3/ 6 patients [50.0\%]; $p=0.3403$ ). Int-1-risk patients with high EPO levels were also more likely to have mutations in any of the 5 genes previously linked to poor prognosis 
a

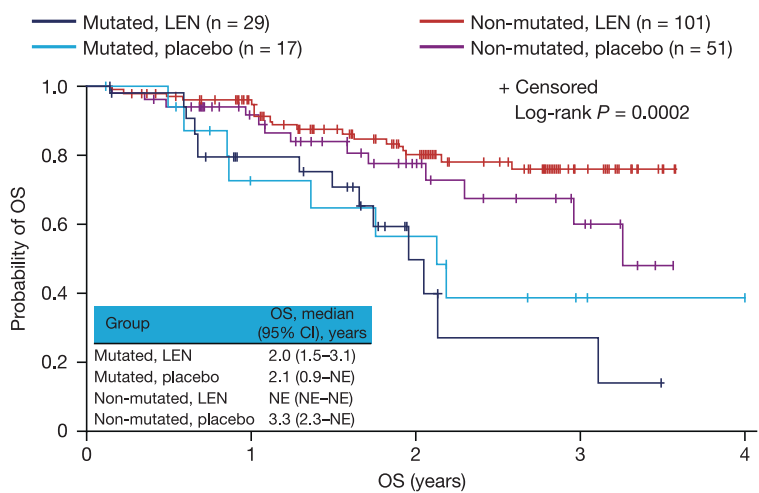

c

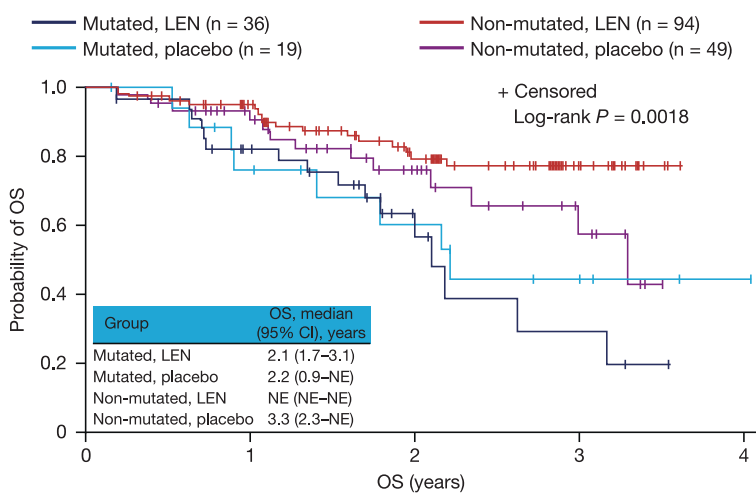

b

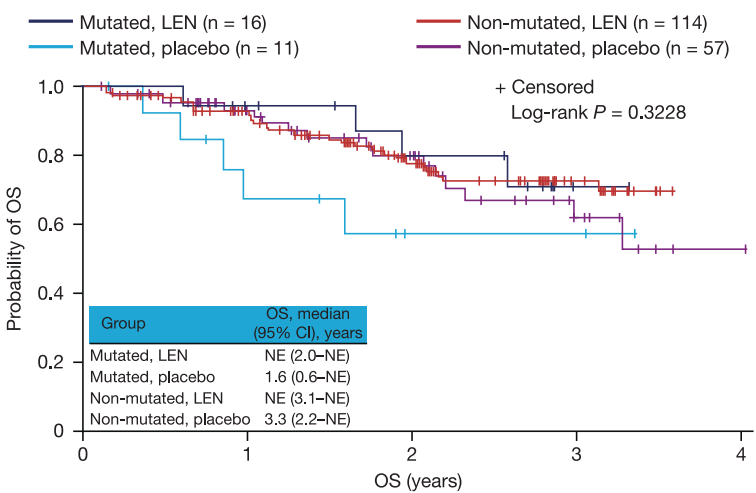

d

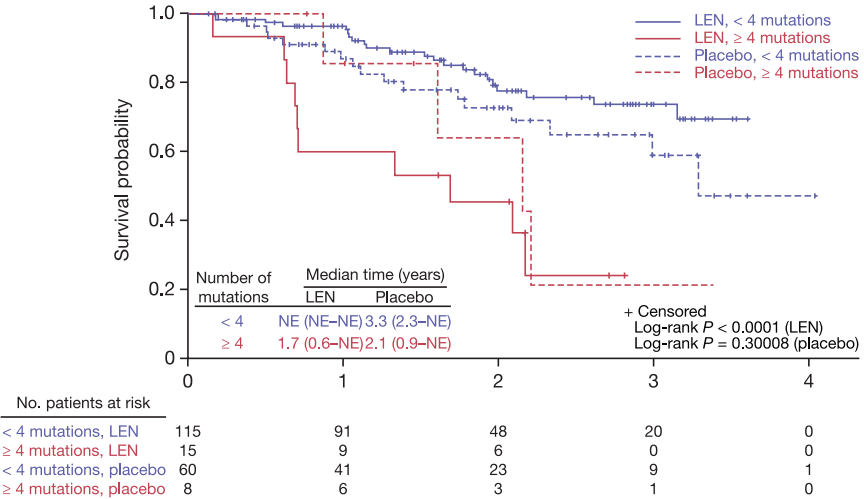

Fig. 2 Overall survival by treatment group and mutation status. For ASXL1 (a), DNMT3A (b), any 1 of 5 genes in the Bejar 5-gene mutation signature (c), and number of mutations (d). CI confidence interval, LEN lenalidomide, NE not estimable, OS overall survival.

(ASXL1, ETV6, EZH2, RUNX1, and TP53) [12] compared to those with low EPO levels (60.6\% vs. 39.4\%; $p=0.0102)$; there was no significant difference for Low-risk patients.

The high prevalence of mutations and mutational profile are consistent with results from a previous analysis of patients with MDS $[1,2,12]$. The effect of ASXL1 mutations on response rate to lenalidomide and OS, regardless of treatment, is consistent with prior reports showing an adverse prognostic effect of $A S X L 1$ mutations [2, 12, 13]. Also, the trend towards improved response to lenalidomide in patients with DNMT3A mutations is consistent with results from a trial of patients with non-del(5q) MDS in which DNMT3A mutations predicted better response [10]. Patients with DNMT3A mutations have higher rates of progression to acute myeloid leukemia (AML) [14]. It may therefore be that the trend towards improved OS in DNMT3A-mutated patients receiving lenalidomide in this study was a result of a reduction in the rate of AML progression.

The association of increased mutation burden with worse OS is consistent with previous reports of a link with shorter leukemia-free survival [1]. Patients with $\geq 4$ mutations were more likely to have a high serum EPO level (>500 mU/ml), a negative prognostic marker associated with a lower probability of response to treatment [8, 15]. High serum EPO level was associated with the presence of mutations in any of 5 poor-prognosis genes (ASXL1, ETV6, EZH2, RUNX1, and TP53) [13] and specifically ASXL1. Taken together with the observed association between ASXL1 mutations and low response rate to lenalidomide, this provides further insight into previous observations that patients with high baseline EPO level have a lower probability of responding to lenalidomide [8].

Limitations of this analysis include the confounding effects of co-mutations on outcomes and the relatively small and heterogeneous group of patients.

A better understanding of how somatic mutations influence lenalidomide response may help identify patients with lower-risk non-del(5q) MDS who are most likely to respond to lenalidomide therapy. Efforts are currently ongoing to incorporate mutations into the IPSS-R to improve its prognostic value [13]. Currently, there are no unequivocal genetic markers of response to non-therapeutic targets, such 
as lenalidomide for lower-risk non-del(5q) MDS. However, our finding that ASXL1 mutations negatively affect outcomes with lenalidomide is relevant, as negative prognostic factors are not always predictive of poor response, as was demonstrated by the contrasting finding for TP53 in MDS patients treated with decitabine [4]. Thus, identifying predictive markers of response to lenalidomide in non-del(5q) MDS patients remains an ongoing challenge.

Acknowledgements This study was sponsored by Celgene, a whollyowned subsidiary of Bristol-Myers Squibb Company, Princeton, NJ, USA. The authors received editorial and writing support provided by Miriam de Boeck, from Excerpta Medica, funded by the sponsor. The authors are fully responsible for all content and editorial decisions.

Author contributions VS, PF, AG, UP, AFL, and AA contributed to the collection of data; VS designed the study; JZ, CW, and KJM performed the statistical analyzes; all authors were involved in the analysis and interpretation of the data, review and revision of the work, and approval of the final version of the manuscript.

\section{Compliance with ethical standards}

Conflict of interest VS has received honoraria from Celgene Corporation, Janssen Pharmaceuticals, Amgen, Astex, Takeda, and Novartis; PF has received research funding and honoraria from Celgene Corporation, Janssen Pharmaceuticals, Jazz Pharmaceuticals, and Otsuka Pharmaceutical; AG is a consultant for and has received honoraria from Celgene Corporation; UP and AFL have received research funding from Celgene Corporation; JZ, CW, KM, CLB, and $\mathrm{KJM}$ are employees of and have equity ownership in Celgene Corporation; AA is a consultant for and has received honoraria from Celgene Corporation and Novartis, has received research funding from Celgene Corporation, and has presented at speakers' bureau for Bristol Myers Squibb, Celgene Corporation, Novartis, and Shire.

Publisher's note Springer Nature remains neutral with regard to jurisdictional claims in published maps and institutional affiliations.

Open Access This article is licensed under a Creative Commons Attribution 4.0 International License, which permits use, sharing, adaptation, distribution and reproduction in any medium or format, as long as you give appropriate credit to the original author(s) and the source, provide a link to the Creative Commons license, and indicate if changes were made. The images or other third party material in this article are included in the article's Creative Commons license, unless indicated otherwise in a credit line to the material. If material is not included in the article's Creative Commons license and your intended use is not permitted by statutory regulation or exceeds the permitted use, you will need to obtain permission directly from the copyright holder. To view a copy of this license, visit http://creativecommons. org/licenses/by/4.0/

\section{References}

1. Papaemmanuil E, Gerstung M, Malcovati L, Tauro S, Gundem G, Van Loo P, et al. Clinical and biological implications of driver mutations in myelodysplastic syndromes. Blood. 2013; 122:3616-27.

2. Haferlach T, Nagata Y, Grossman V, Okuno Y, Bacher U, Nagae $\mathrm{G}$, et al. Landscape of genetic lesions in 944 patients with myelodysplastic syndromes. Leukemia. 2014;28:241-7.

3. Bejar R, Lord A, Stevenson K, Bar-Natan M, Pérez-Ladaga A, Zaneveld $\mathrm{J}$, et al. TET2 mutations predict response to hypomethylating agents in myelodysplastic syndrome patients. Blood. 2014;124:2705-12.

4. Welch JS, Petti AA, Miller CA, Fronick CC, O'Laughlin M, Fulton RS, et al. TP53 and decitabine in acute myeloid leukemia and myelodysplastic syndromes. $\mathrm{N}$ Engl J Med. 2016;375:2023-36.

5. Montalban-Bravo G, Takahashi K, Patel K, Wang F, Xingzhi S, Nogueras GM, et al. Impact of the number of mutations in survival and response outcomes to hypomethylating agents in patients with myelodysplastic syndromes or myelodysplastic/ myeloproliferative neoplasms. Oncotarget. 2018;9:9714-27.

6. Kosmider O, Passet M, Santini V, Platzbecker U, Andrieu V, Zini $\mathrm{G}$, et al. Are somatic mutations predictive of response to erythropoiesis stimulating agents in lower risk myelodysplastic syndromes? Haematologica. 2016;101:e280-3.

7. Fenaux P, Platzbecker U, Mufti GJ, Garcia-Manero G, Buckstein $\mathrm{R}$, Santini V, et al. Luspatercept in patients with lower-risk myelodysplastic syndromes. N Engl J Med. 2020;382:140-51.

8. Santini V, Almeida A, Giagounidis A, Gröpper S, Jonasova A, Vey N, et al. Randomized phase III study of lenalidomide versus placebo in RBC transfusion-dependent patients with lower-risk non-del $(5 q)$ myelodysplastic syndromes and ineligible for or refractory to erythropoiesis-stimulating agents. J Clin Oncol. 2016;34:2988-96.

9. Toma A, Kosmider O, Chevret S, Delaunay J, Stamatoullas A, Rose C, et al. Lenalidomide with or without erythropoietin in transfusion-dependent erythropoiesis-stimulating agent-refractory lower-risk MDS without $5 \mathrm{q}$ deletion. Leukemia. 2016;30:897-905.

10. Chesnais V, Renneville A, Toma A, Lambert J, Passet M, Dumont F, et al. Effect of lenalidomide treatment on clonal architecture of myelodysplastic syndromes without $5 \mathrm{q}$ deletion. Blood. 2016;127:749-60.

11. Cheson BD, Greenberg PL, Bennett JM, Lowenberg B, Wijermans PW, Nimer SD, et al. Clinical application and proposal for modification of the International Working Group (IWG) response criteria in myelodysplasia. Blood. 2006;108:419-25.

12. Bejar R, Stevenson K, Abdel-Wahab O, Galili N, Nilsson B, Garcia-Manero G, et al. Clinical effect of point mutations in myelodysplastic syndromes. N Engl J Med. 2011;364:2496-506.

13. Bejar R, Papaemmanuil E, Haferlach T, Garcia-Manero G, Maciejewski JP, Sekeres MA, et al. Somatic mutations in MDS patients are associated with clinical features and predict prognosis independent of the IPSS-R: analysis of combined datasets from the International Working Group for Prognosis in MDS-Molecular Committee. Blood. 2015;126:907.

14. Walter MJ, Ding L, Shen D, Shao J, Grillot M, McLellan M, et al. Recurrent DNMT3A mutations in patients with myelodysplastic syndromes. Leukemia. 2011;25:1153-8.

15. Platzbecker U, Germing U, Götze KS, Kiewe P, Mayer K, Chromik $\mathbf{J}$, et al. Luspatercept for the treatment of anaemia in patients with lower-risk myelodysplastic syndromes (PACEMDS): a multicentre, open-label phase 2 dose-finding study with long-term extension study. Lancet Oncol. 2017;18:1338-47. 